\title{
Hacia una propuesta de selección léxica en manuales de español como lengua extranjera de nivel $\mathrm{A} 1^{*}$
}

Towards a lexical selection proposal in Spanish as a foreign language manuals, A1 level

\author{
Ángel Huete García \\ Universitat Rovira i Virgili, \\ Departament de Filologies Romàniques \\ Antoni Nomdedeu Rull \\ Universitat Rovira i Virgili, \\ Departament de Filologies Romàniques
}

\section{Resumen}

Las editoriales españolas de español como lengua extranjera (ELE) no especifican en sus manuales el origen del léxico que se recoge en ellos ni el criterio para su selección. Esta omisión es importante si se considera el papel de los corpus textuales en la enseñanza de segundas lenguas de las últimas décadas. Este estudio pretende sentar las bases para determinar y caracterizar el léxico de la alimentación en español correspondiente al nivel A1.
A partir de los datos recopilados del análisis de ocho manuales de ELE, se realiza una comparación crítica con base en los criterios de selección del léxico básico para obtener el vocabulario fundamental del español, tales como la disponibilidad léxica o la frecuencia, además de contrastar esta información con los documentos de referencia (PCIC y MCER) y corpus. Al final, se ofrece una propuesta de selección léxica de la alimentación para el nivel A1.

Este artículo se enmarca en el proyecto de investigación Diccionario de español para aprendices sino hablantes (fase primera) (Ref. FFI2016-76417-P) del Grupo de Investigación en Lexicografía y Enseñanza de Lenguas Extranjeras (Lexele) de la Universidad Rovira i Virgili, Grupo Consolidado de la Generalitat de Catalunya (SGR 2014 SGR 1288). 
Palabras clave: enseñanza de léxico de ELE; manuales de ELE; frecuencia léxica; disponibilidad léxica

\begin{abstract}
The Spanish publishers of Spanish as a lected from the analysis of eight SFL manuForeign Language (SFL) usually do not specify the origin of the words that they compile in their books, or the criteria used for collecting their lexicon. These omissions become more relevant if we consider the importance of the Corpus in the context of second-language teaching over the past ten years. In this regard, this study aims to identify and characterize the food lexicon als served to conduct a critical comparison based on the selection criteria of basic lexicon to obtain the essential Spanish vocabulary, such as lexicon availability or frequency, besides contrasting this information versus reference documents (PCIC and CEFR). As an end result, this study includes a proposal for the selection of food lexicon to be used in the A1 level.
\end{abstract} for Spanish in the A1 level. The data col-

Keywords: SFL lexicon teaching; sFL Manuals; lexicon frequency; lexicon availability 


\section{Introducción}

La selección del léxico necesario para el aprendizaje de una L2 es una de las cuestiones más debatidas de los últimos años en las investigaciones sobre enseñanza del vocabulario, ya que los criterios para seleccionar el léxico fundamental son a menudo heterogéneos y subjetivos. Con la incursión de las nuevas tecnologías en el contexto de la enseñanza y aprendizaje de segundas lenguas se ha conseguido avanzar sustancialmente en este aspecto, sobre todo, en la obtención de datos sobre los usos léxicos de los hablantes, mediante criterios como la frecuencia o disponibilidad de las palabras.

A pesar de estos avances, aún no existe una regla universal que categorice el léxico en español en los diferentes umbrales de aprendizaje, a pesar de que los manuales de español como lengua extranjera (ELE) implícitamente sí lleven a cabo dicha separación. No obstante, se documentan algunos intentos de clasificar el léxico por niveles, como el de Ferrando (2012) y el de Ji (2017). Ferrando (2012: 10) presentó "una propuesta para la compilación de un diccionario combinatorio destinado a la enseñanza-aprendizaje de E/LE por niveles" y Ji (2017) estableció recientemente criterios para agrupar los términos del futbol por niveles con el doble objetivo de, por un lado, elaborar un glosario bilingüe que pueda facilitar el aprendizaje del léxico futbolístico a los estudiantes chinos de ELE interesados en desarrollar su labor profesional en dicho ámbito y, por el otro, elaborar una unidad didáctica (nivel B1). Ambos autores realizan sus propuestas ante la escasez de datos que impide agrupar conjuntos de palabras para cada uno de los niveles.

En este contexto nos planteamos la pregunta de investigación siguiente: ¿qué criterios se siguen en los manuales de ELE para seleccionar el léxico que incluyen en sus actividades? Nuestra hipótesis es que en los manuales españoles actuales de ELE no se especifican estos criterios ni se tienen en cuenta los datos de frecuencia y disponibilidad léxica proporcionados por los diversos corpus existentes. A este respecto, pensamos, como Benítez, que "son pocos los materiales que se han creado teniendo en cuenta lo que aquí defendemos, ${ }^{1}$ sólo [sic]

1 Se refiere al estudio ordenado del vocabulario, seleccionado cuidadosamente teniendo en cuenta su frecuencia de aparición y su utilidad. 
conocemos el manual que sigue la metodología audiovisual de Rojo, Rivenc y Ferrer, titulado Vidas y diálogos de España, publicado en París por Didier" (2009: 11). ${ }^{2}$

A partir de nuestra pregunta de investigación y para corroborar nuestra hipótesis nos proponemos alcanzar el objetivo siguiente: presentar una propuesta de selección léxica centrada en el ámbito de la alimentación y correspondiente al nivel A1. Hemos acotado el objeto de análisis con el fin de poder abarcar exhaustivamente una parcela de entre todos los centros de interés existentes en los manuales de ELE. Para llegar a dicha selección léxica hemos seguido la siguiente metodología: 1) buscar referencias a los criterios seguidos para seleccionar el léxico en los manuales de ELE analizados; 2) discutir las nociones específicas sobre el léxico de la alimentación contenidas en el Plan Curricular del Instituto Cervantes (PCIC) y 3) contrastar dicho léxico en ocho manuales de ELE con datos sobre frecuencia obtenidos en el Corpus de Referencia del Español Actual (CREA $)^{3}$ y disponibilidad léxica. Nuestro objeto de estudio no tiene que ver con el análisis de cómo enseñar ELE, ${ }^{4}$ sino con qué léxico enseñar ELE en función del nivel de aprendizaje específico.

En este estudio presentamos un breve estado de la cuestión sobre la selección del léxico (§2), una descripción y caracterización — tanto cualitativa como cuantitativa - de los manuales de ELE pertenecientes a las editoriales más importantes de España y del léxico en ellos recopilado $(\$ 2.1,2.2)$. Para ello

2 Con respecto a este manual de 1968, los autores se basaron en un corpus de 1200000 palabras procedentes de dos fuentes: por un lado, 800000 procedían de la transcripción de conversaciones reales tomadas en 130 ciudades y pueblos de España, entre individuos pertenecientes a 110 profesiones y 7 niveles de educación diferentes; por el otro, 400000 de una encuesta realizada mediante cuestionarios elaborados a partir de la técnica de centros de interés, para determinar el vocabulario disponible. Con estos datos, y en el contexto de la metodología audiovisual, elaboraron diálogos, situaciones gramaticales, dictados y textos de lectura.

3 Sabemos de la existencia de otra lista de frecuencias importantes, como A frequency dictionary of Spanish: Core vocabulary for learners de Mark Davies (2006) o el Corpus del español del mismo autor, http://www.corpusdelespanol.org/, pero, para los objetivos de este estudio, preferimos emplear documentos considerados de referencia, como en este caso el CREA.

4 En un estudio reciente, Cruz (2015) proporciona numerosos y útiles materiales para enseñar y aprender léxico con tecnologías: interfaces lexicográficas en línea, corpus, materiales didácticos en línea, etcétera. 
se contrastaron los principales documentos de referencia a este respecto - el PCIC y el Marco Común Europeo de Referencia para las Lenguas: aprendizaje, enseñanza, evaluación (MCER) del Consejo de Europa- (\$2.3), así como una recopilación de datos cuantitativos ofrecidos por distintos corpus (CREA y Dispolex, §3). Por último, ofrecemos una propuesta de selección del léxico fundamental en español de la alimentación correspondiente al nivel A1 a partir de la consideración de los datos cuantitativos analizados y del criterio de la limitación objetiva corregida (De Grève \& Van Passel, 1971): frecuencia, eficacia y productividad del léxico (\$4).

\section{La selección del léxico}

En este apartado nos referimos brevemente a algunas cuestiones básicas relativas a la frecuencia y disponibilidad léxicas como criterios que deberían guiar la selección del léxico y a los lineamientos marcados por el MCER y el PCIC para este mismo fin.

\subsection{Los corpus y la necesidad de contar con datos cuantificables}

Los factores útiles a la hora de seleccionar el léxico de una lengua son varios: frecuencia, disponibilidad, rentabilidad, utilidad, dificultad de las voces o factores culturales, entre otros. En este estudio nos centramos en el análisis de elementos que pueden representarse con una relativa mayor objetividad, es decir, datos de frecuencia y disponibilidad de las palabras, con el fin de compararlos con la selección léxica realizada por ocho manuales de ELE.

Una de las constantes expresadas en la bibliografía sobre los estudios de lingüística aplicada a la enseñanza de lenguas extranjeras es la necesidad de abordar cuestiones como la selección léxica a partir de criterios lingüísticos lo más objetivos posible. Dichos datos pueden complementarse con los ofrecidos por los listados de frecuencias y de disponibilidad léxica. A este respecto, contamos con datos representativos desde hace unos 50 años que supusieron un cambio importante para diversos sectores de la lingüística - la lexicografía, la traducción y la didáctica de lenguas extranjeras. El desarrollo tecnológico de los medios informáticos ha conllevado, más recientemente, que el interés en la didáctica de len- 
guas extranjeras se centre de nuevo en el léxico que hay que enseñar y aprender, ya que herramientas como los corpus permiten analizar cuantitativamente el léxico, lo que posibilita plantear un análisis ulterior basado en aspectos cognitivos importantes para el aprendizaje, como la situación (Ávila, 2010).

El estudio del léxico por frecuencias, con el objetivo de crear manuales de enseñanza de una lengua extranjera, se remonta al año 1954 con la publicación del Français Fondamental, proyecto que tenía como objetivo seleccionar el léxico del francés en un nivel básico. Sin embargo, la clasificación no contaba con palabras cotidianas y fácilmente reconocibles como autobus, métro y timbre, ya que, para los hablantes, el uso de estas voces estaba determinado por el tema de la conversación o del texto (Bartol, 2006). Por consiguiente, se infirió que para activar estas palabras del lexicón mental se necesitaba hacer uso de pruebas asociativas. Este tipo de proyectos estadísticos aparecieron en español también en esta década, como es el caso del Vocabulario usual, común y fundamental de García Hoz (1953) con un total de 15000 palabras.

La frecuencia de las palabras constituye uno de los criterios principales en la selección del léxico básico de una lengua. Desde los años sesenta del siglo pasado, contamos con varios corpus y listados de frecuencias que pueden aprovecharse para la elaboración de materiales didácticos de ELE: Frequency dictionary of Spanish words de Juilland y Chang-Rodríguez (1964), Vocabulario del español hablado de Márquez (1975), El desarrollo del vocabulario: diccionario de frecuencias de Justicia (1995), Cumbre: Corpus lingüístico del español contemporáneo: fundamentos, metodología y aplicaciones de Sánchez, Sarmiento, Cantos y Simón (2001), Frecuencias léxicas del español coloquial: análisis cuantitativo y cualitativo de Terrádez (2001), A frequency dictionary of Spanish: Core vocabulary for learners de Davies (2006) y el CREA de la Real Academia Española (RAE).

Para solventar los límites del método de la frecuencia, pueden aprovecharse los resultados que proporcionan los estudios de disponibilidad léxica, por medio de los cuales se pretende recoger y analizar qué palabras sería capaz de usar un hablante en determinados temas de comunicación. A este respecto, los investigadores del Français Fondamental, dirigidos por Gougenheim, establecieron un nuevo criterio de selección del léxico: la disponibilidad léxica, esto es el "caudal léxico utilizable en una situación comu- 
nicativa dada" (López Morales, 1995: 245). Este criterio responde a una voluntad de los autores franceses por perfeccionar las deficiencias que ofrecían los listados de frecuencias utilizados hasta el momento para seleccionar el léxico. Algunos de los resultados de los diferentes estudios de disponibilidad léxica publicados en los últimos años, sin bien son aún escasos, pueden observarse en el portal Dispolex.

Con la suma del léxico básico — que recoge las palabras más frecuentes mediante la aplicación de fórmulas de frecuencia, dispersión y uso y con independencia del tema que se trate- y del disponible se obtiene el léxico fundamental de una comunidad de habla (López Morales, 1995). Estas unidades pueden ser identificadas gracias a los corpus textuales, cuya importancia en el marco de la enseñanza de lenguas se explica porque permiten la interacción con los materiales y manuales de aprendizaje (Alonso, 2007: 12-13, a partir de las aportaciones de McCullough 2001). Y así, como afirma Santos en referencia al español: "establecer un VF [vocabulario fundamental] del español supone, pues, una alternativa más completa y que se puede orientar directamente a la enseñanza-aprendizaje de ELE" (2017: 116).

A pesar de que la selección de los contenidos del corpus para establecer un léxico fundamental puede realizarse teniendo en cuenta distintos criterios, todo parece indicar que el mejor procedimiento, por su objetividad, es el de la frecuencia. El principal problema con el que se encuentran los manuales de ELE en la actualidad es que la correspondencia de los contenidos seleccionados y los datos léxicos referidos a esos contenidos —extraídos de los corpus- demuestran que "el input ofrecido en los libros se ha manipulado en exceso" (Alonso, 2007: 18-19). Cabría añadir que, en realidad, se ha manipulado solo si se ha consultado previamente. De hecho, el análisis que realizamos de los manuales pone de manifiesto, tal y como declaran Rodríguez y Muñoz (2009: 9), que "en ocasiones, estos parten de premisas aleatorias que causan una sensación de inconcordancia y poca sintonía con la realidad léxica del idioma”.

Por tanto, aunque en los últimos años se ha experimentado un desarrollo importante de la linguíística de corpus aplicada a la creación de materiales y manuales de enseñanza de ELE, todavía quedan por superar algunas barreras tradicionalistas en cuanto a los criterios de selección del léxico. El uso de corpus textuales puede ayudar a mejorar los materiales de enseñanza siempre y cuando el vaciado 
de contenidos aproveche sus posibilidades, como es el hecho de presentar el léxico contextualizado y a partir de las necesidades e intereses del estudiante.

\subsection{Criterios generales para seleccionar el léxico}

La complejidad del léxico de cualquier lengua produce que la selección léxica, establecida con un fin descriptivo o didáctico, sea una tarea difícil de completar, ya que los elementos lingüísticos y extralingüísticos que intervienen son muchos. Sin embargo, desde los años setenta ha habido algunos intentos de clasificar y establecer los principales criterios de selección léxica.

De Grève y Van Passel (1971: 106-124) postularon tres métodos mediante los que puede seleccionarse el vocabulario que aparece en los manuales de enseñanza: 1) limitación subjetiva, 2) limitación estrictamente objetiva y 3) limitación objetiva corregida. La limitación subjetiva, el criterio más utilizado a lo largo de los años, se basa exclusivamente en la intuición del profesor o del autor de manuales. Si bien la intuición de un especialista puede ser en muchas ocasiones acertada, este método subjetivo es el que ha cosechado más problemas, ya que no es suficiente para justificar una selección que debería plantearse de inicio como objetiva. Con todo, aceptamos la idea de usar la apreciación intuitiva basada en la experiencia del docente cuando el usuario se haya definido con exactitud. En este caso, la intuición del docente es enormemente funcional, puesto que la estrecha colaboración con el experto aporta numerosas soluciones. En los últimos años dentro del ámbito de la lexicografía, se ha propuesto para la selección del léxico el concepto de proscripción, que supone indicar claramente qué variante se prefiere o recomienda entre diferentes candidatos ortográficos, fonéticos o flexivos:

If a lexicographer chooses to be proscriptive, the way he deals with the results of the empirical analysis differs from that of being descriptive. The lexicographer does not stop after having informed the user about language use, he also wants to tell the user which variant he should choose if there is more than one 
possibility. This lexicographical method of presentation can be called proscription, in practice it is a selective description (Bergenholtz, 2003: 77). ${ }^{5}$

En el ámbito relativo a la elaboración de materiales didácticos de ELE, la proscripción podría aplicarse en la selección léxica.

En lo que respecta al segundo de los criterios, la limitación estrictamente objetiva, el léxico responde a una selección basada en la utilidad que, en otras palabras, alude al vocabulario de uso más frecuente. En este caso se seleccionan las palabras que se puedan utilizar "en un mayor número de circunstancias diferentes" (Benítez, 2009: 11). No obstante, el criterio basado en la frecuencia plantea problemas que imposibilitan una selección léxica completamente objetiva. En primer lugar, no contempla la totalidad del léxico — recordemos las diferencias entre léxico frecuente y léxico usual observadas en el Français Fondamental. En segundo lugar, los estudios y recopilaciones de léxico frecuente no consideran la realidad lingüística de todas sus variedades dialectales. En los corpus se recopilan textos mayoritariamente en el español de España —el $R e$ cuento de vocabulario español (1952) es un ejemplo de ello-, mientras que las diferentes variantes del español americano no suelen contar con una presencia representativa (Salazar, 2004). De acuerdo con Alvar, "las listas de frecuencias no son la solución de los problemas de la enseñanza del léxico, aunque su utilidad es evidente" (2005: 21).

El tercero de los criterios, limitación objetiva corregida, no solamente tiene en cuenta la frecuencia, sino también la eficacia y la productividad del léxico. Se entiende en este caso por palabras eficaces aquellas que pueden utilizarse en contextos diferentes (p. ej., tren es más eficaz que talgo o cercanías) y por palabras productivas, "las que pueden dar lugar a otras al añadirle ciertos formantes” (p. ej., zapato, zapatero y zapatería suponen una cadena de palabras productivas) (Benítez, 2009: 11). Este criterio es el que cuenta con un mayor consenso en la actualidad porque confluyen en él diferentes variables importantes

5 "Si un lexicógrafo elige ser proscriptivo, la forma en que trata los resultados del análisis empírico difiere de la de ser descriptivo. El lexicógrafo no se detiene después de haber informado al usuario sobre el uso del idioma, también quiere decirle al usuario qué variante debe elegir si hay más de una posibilidad. Este método lexicográfico de presentación puede denominarse proscripción, en la práctica es una descripción selectiva" (traducción nuestra). 
que no excluyen palabras básicas, como sí ocurre si solamente se considera la frecuencia. Sin embargo, este criterio no suele tomarse en cuenta en la realización de manuales de ELE, lo que confirmamos tanto en los datos proporcionados en las obras como en los que mostraremos en este estudio ( $\$ 3)$.

No existe consenso sobre la utilidad de la clasificación, ya tradicional, de De Grève y Van Passel (1971). Por ejemplo, López Pérez señala que dicha categorización "no resulta adecuada para una competencia en temas especializados, como pueden ser los de las distintas asignaturas del currículo", además de que "no entra en detalles sobre los factores que motivan la elección" (2013: 160). En nuestro caso, en cambio, sí podría ser de utilidad al tratarse de un ámbito general, ya que este no tiene las limitaciones que sí presentaría un curso para especialistas en cocina porque el léxico vendría delimitado por otros factores extralingüísticos que no se contemplan siguiendo ese criterio.

Por otro lado, la clasificación propuesta por Bogaards (1994), inspirada en la de Scherfer (1985), establece dos factores que intervienen en el proceso de selección del vocabulario: criterios pedagógicos y criterios lingüísticos — frecuencia, medidas subjetivas tales como disponibilidad o familiaridad, valencia léxica y situaciones, nociones y funciones. Con todo, indica que "le seul critère réel de sélection est l'utilité" [el verdadero criterio de selección es la utilidad] (Bogaards, 1994: 111) y que es fundamental "savoir à qui peuvent servir les mots pour les apprenants d'une langue étrangère" [saber quién puede usar las palabras para los alumnos de un idioma extranjero] (Bogaards, 1994: 112). Por tanto, considera necesario dar respuesta a las dos preguntas siguientes: ¿cuáles son las tareas que el aprendiz de una lengua extranjera tendrá que ejecutar en las situaciones en las que se va a encontrar?, ¿cómo se le puede proporcionar material léxico que le permitirá expresarse y comprender los mensajes? Por lo que respecta a la frecuencia, menciona la naturaleza de las palabras frecuentes, analiza la relación entre frecuencia y valor de comunicación y la relación entre léxico y estadística. De las medidas subjetivas, tiene en cuenta la disponibilidad, la familiaridad y las estimaciones subjetivas de la frecuencia. Dentro de la valencia léxica — criterio de uso que mide las palabras que tienen la capacidad de reemplazar otras palabras - , trata la inclusión, la extensión, la composición y la definición. Y finalmente, bajo "Situaciones, nociones y funciones", se refiere, en aras de conocer las necesidades de los aprendices de una L2, a "analyser le langage in- 
dispensable pour remplir les fonctions linguistiques nécessaires en rapport avec les notions qui apparaissent normalement dans des situations bien définies"6 (1994: 137).

En definitiva, De Grève y Van Passel (1971) llegaron a la conclusión de que "se impone la necesidad de enseñar una lengua extranjera partiendo de un vocabulario escogido según criterios establecidos científicamente" (1971: 133). Consideramos que en esta tendencia deben basarse los manuales de ELE en lo que respecta a la selección del léxico que en ellos se incluyen.

Por otra parte, para el análisis ordenado del vocabulario según su frecuencia y utilidad, la disponibilidad léxica es para algunos autores el criterio más eficiente al respecto (Benítez, 2009). En cambio, estudiosos como Salazar (2004), quien expone algunas de las dificultades a las que se enfrentan los estudios de disponibilidad léxica, proponen que se lleve a cabo una selección "que contemple de forma combinada los tres" (2004: 261). La propuesta de Salazar se explica porque los estudios de disponibilidad léxica tratan de descifrar "una realidad no directamente observable" (2004: 259), que no es otra que la del lexicón mental de los hablantes.

En la actualidad los resultados del Proyecto Panhispánico de Léxico Disponible (Dispolex) del español —que tiene como objetivo determinar el léxico fundamental del español mediante la colaboración de todos los proyectos en marcha en las distintas zonas hispanohablantes-, no son aún representativos y, por lo tanto, no podemos basarnos en ellos de una manera general. En lo que respecta al ámbito considerado en este estudio, alimentación, contamos con datos proporcionados por José Antonio Bartol Hernández relativos a las comidas y bebidas de la provincia de Soria y con los facilitados por José Ramón Gómez Molina, de Valencia.

Asimismo, tal y como apunta Paredes, hay propuestas actuales que "abogan por una mayor consonancia con las indicaciones contenidas en el MCER" (2014: 57). En el ámbito del español tenemos como referencia las "Nociones específicas" del PCIC, que analizaremos a continuación junto con las indicaciones del MCER.

6 “[A]nalizar el lenguaje indispensable para cumplir las funciones lingüísticas necesarias en relación con las nociones que normalmente aparecen en situaciones bien definidas." 


\subsection{Criterios para la selección del léxico en el MCER y el PCIC}

En este subapartado analizamos los criterios que siguen el MCER y el PCIC para la selección del léxico con el propósito de observar el grado de fidelidad de los manuales con ambos documentos de referencia.

Dentro de las "escalas ilustrativas para la gradación del conocimiento del vocabulario" (Consejo de Europa, 2002: 109), observamos que el nivel A1 configura un umbral bastante cerrado en comparación con otras escalas del MCER al tratarse de un vocabulario simple y muy concreto, como se observa en el Cuadro 1. No obstante, ese repertorio básico puede verse alterado por cuestiones culturales y geográficas. La definición de estas escalas de nivel señala "la relación entre el léxico y las situaciones de comunicación” (Baralo, 2007: 386) y permite diferenciar niveles.

\begin{tabular}{|c|c|c|}
\hline & RIQUEZA DE VOCABULARIO & DOMINIO DE VOCABULARIO \\
\hline $\mathrm{A} 1$ & $\begin{array}{l}\text { Tiene un repertorio básico de palabras } \\
\text { y frases aisladas relativas a situaciones } \\
\text { concretas. }\end{array}$ & No hay descriptor disponible. \\
\hline
\end{tabular}

En cuanto a la selección léxica propiamente dicha, el MCER (Consejo de Europa, 2002: 149-150) propone cuatro formas posibles de selección:

a) elegir palabras y frases clave:

- en áreas temáticas necesarias para la consecución de tareas comunicativas adecuadas a las necesidades de los alumnos;

- que encarnen la diferencia cultural y, en su caso, los valores y creencias importantes compartidos por el grupo o los grupos sociales cuya lengua se está enseñando;

b) seguir principios léxico-estadísticos que seleccionen las palabras más frecuentes en recuentos generales y amplios o las palabras que se utilizan para áreas temáticas delimitadas;

c) elegir textos (auténticos) hablados y escritos y aprender o enseñar todas las palabras que contienen; 
d) no realizar una planificación previa del desarrollo del vocabulario, más bien permitir que se desarrolle orgánicamente en respuesta a la demanda del alumno cuando éste se encuentre realizando tareas comunicativas.

En su revisión de esta propuesta, Bartol (2010: 89) considera que las dos últimas opciones, incisos c y d, no son verdaderas formas de selección léxica. De hecho, un análisis profundo de lo expuesto induce a considerar que la opción a se interprete como una selección subjetiva que confía en la intuición de los profesores o autores de materiales, mientras que el caso b se correspondería con una selección objetiva basada en corpus por medio del uso de métodos estadísticos de análisis.

Desde el PCIC se matiza que la clasificación que se ofrece es arbitraria y orientativa porque, como se verá más adelante con la selección léxica del ámbito de la alimentación, esa lista no puede concebirse como un conjunto cerrado, ya que depende totalmente de "las necesidades y expectativas del alumno", así como "de las distintas variedades de la lengua en el riquísimo acervo del español y en relación siempre con el entorno geográfico, social y cultural en el que se desarrolle el proceso de enseñanza y aprendizaje" (PCIC, "Nociones específicas. Introducción"). Además, todo el criterio de selección léxica seguido por el PCIC está basado en la variedad centro-norte peninsular española, hecho que dificulta las posibles incorporaciones del léxico de Hispanoamérica, cuya realidad es diferente en términos de frecuencia y disponibilidad. En este mismo sentido, el inventario de las "Nociones específicas" es también una base de referencia de selección del léxico y de su distribución por niveles.

Con todo, considerando los objetivos de esta investigación, nos centramos en la metodología empleada para seleccionar el léxico por niveles a partir de un tema. En este sentido, el PCIC ha tenido en cuenta los "Objetivos generales" para los niveles de referencia del español y las indicaciones del proyecto Can Do de la Association of Language Testers in Europe (ALTE, 2002).

Los objetivos generales de los niveles de descripción de la competencia linguiística presentan desde los años setenta del siglo pasado "una línea de continuidad clara con el MCER" (Instituto Cervantes, 2006: "Objetivos generales. Introducción”). Además, se ha de tener en cuenta, tal y como se explica en la introduc- 
ción a estos objetivos, que la información ofrecida es, a grandes rasgos, para que el usuario se haga una idea del alcance de cada uno de los niveles "sin detallar en exceso los aspectos que se identifican ni aportar un análisis técnico de las categorías que se necesitan para elaborar los objetivos más concretos de los programas de los cursos de español” (Instituto Cervantes, 2006: “Objetivos generales. Introducción”). Estas primeras declaraciones demuestran que el PCIC no espera aportar ningún dato concreto sobre la selección léxica. Toda la definición de los objetivos se ha realizado a partir de tres dimensiones de perspectiva del alumno: alumno como agente social, como hablante intercultural y como aprendiente autónomo. En este sentido, todas las aportaciones de este apartado se centran en el desarrollo de las capacidades del alumno en el proceso de aprendizaje en situaciones comunicativas bajo el rol de agente social.

Así pues, los objetivos generales no proporcionan criterios de selección, sino pautas de comportamiento lingüístico del alumno en situaciones sociales. Estos objetivos son variables en función de "las características de cada situación particular de enseñanza y aprendizaje" (Instituto Cervantes, 2006: “Objetivos generales. Introducción”), de manera que no se trata de listas cerradas, sino que los contenidos, en nuestro caso léxicos, vendrían determinados por esas situaciones particulares.

Somos conscientes de que los niveles de referencia para el español no proporcionan un listado de unidades léxicas recogidas bajo el título Léxico sino más bien un inventario de nociones específicas y que para llevar a cabo el inventario han tenido en cuenta las versiones en inglés y en francés y el nivel umbral (PCIC). De este modo, se ha realizado un inventario con veinte temas. De entrada, podemos afirmar que el hecho de llevar a cabo una clasificación de este tipo supone: 1) haber realizado una clasificación muy concreta del mundo que, como cualquier clasificación de esta naturaleza, es arbitraria; 2) que una misma unidad léxica puede aparecer en dos temas distintos y que no son inventarios cerrados, y 3) que la variedad del español elegida es la del centro-norte peninsular española y, por tanto, con las características idiosincrásicas de España. Con referencia a este último aspecto, en un curso de español llevado a cabo en una zona cuya variedad dialectal sea distinta, la elección de las unidades léxicas debería ser, lógicamente, distinta. 
En cuanto al método de selección de las unidades léxicas por tema y por nivel, en el apartado "Nociones específicas. Introducción" del PCIC se indica que

además de los objetivos generales de los Niveles de referencia para el español, se han tenido en cuenta las indicaciones del proyecto Can Do de ALTE y los ámbitos que señala el Marco común europeo de referencia para las lenguas: aprendizaje, enseñanza, evaluación al desarrollar la categoría «contexto de uso»: personal, público, profesional y educativo, así como los distintos parámetros que se presentan en relación con estos ámbitos: lugares, instituciones, personas, objetos, acontecimientos, acciones y textos

En lo que respecta a los criterios seguidos para la selección de dichas unidades léxicas, en el PCIC ("Nociones específicas. Introducción”) se alude a la intuición del docente basada en su experiencia: "Como criterios de selección, siempre partiendo de la apreciación intuitiva basada en la experiencia docente, han primado la frecuencia y la rentabilidad comunicativa". Por tanto, son claros los criterios en los que podemos basarnos a la hora de abordar el análisis de la selección de las unidades léxicas que ha realizado el PCIC y, en consecuencia, la base, en teoría, de gran parte de los manuales de ELE de los últimos diez años: frecuencia y rentabilidad. Con todo, no se facilitan datos sobre los índices consultados ni sobre las decisiones tomadas a este respecto.

En el PCIC también se hace referencia a una cuestión fundamental como es el registro escogido: "También se ha considerado el registro y, de hecho, en los niveles superiores se han incorporado unidades léxicas del registro coloquial y del formal, con el fin de mejorar la precisión y la adecuación comunicativa del alumno que ya domina un registro neutro" ("Nociones específicas. Introducción”). En la realidad, el nivel predominante en las diferentes fases del proceso de enseñanza-aprendizaje de ELE suele ser el neutro. Se entiende aquí por registro neutro aquel que se encuentra a caballo entre lo informal y lo formal, de manera que su alcance puede cubrir las necesidades comunicativas específicas de ambos registros. Por su parte, el proyecto Can Do es un programa de investigación a largo plazo llevado a cabo por la ALTE que tiene como objetivo principal "crear un marco de niveles clave de actuación lingüística dentro del cual se puedan describir exámenes objetivamente" (Instituto Cervantes, 2006: Anexo D). El proyecto, 
que se conoce en español como Puede hacer, tiene como finalidad específica "desarrollar y validar un conjunto de escalas relacionadas con la actuación donde se describa lo que los alumnos son realmente capaces de hacer en la lengua extranjera" (Instituto Cervantes, 2006: Anexo D). Estas especificaciones de capacidad lingüística, que suman un total de cuatrocientas, son multilingües, se han traducido a doce lenguas ${ }^{7}$ y se encuentran repartidas en tres grandes áreas: sociedad y turismo, trabajo y estudio. No analizaremos cómo se elaboró todo el proceso del estudio, ya que escaparía de los límites y objetivos marcados en este estudio, pero sí establecemos una comparación con los textos de referencia, PCIC y MCER. A este respecto, en la Figura 1 puede observarse la estructura de niveles de dominio de una lengua propuesta en el proyecto.

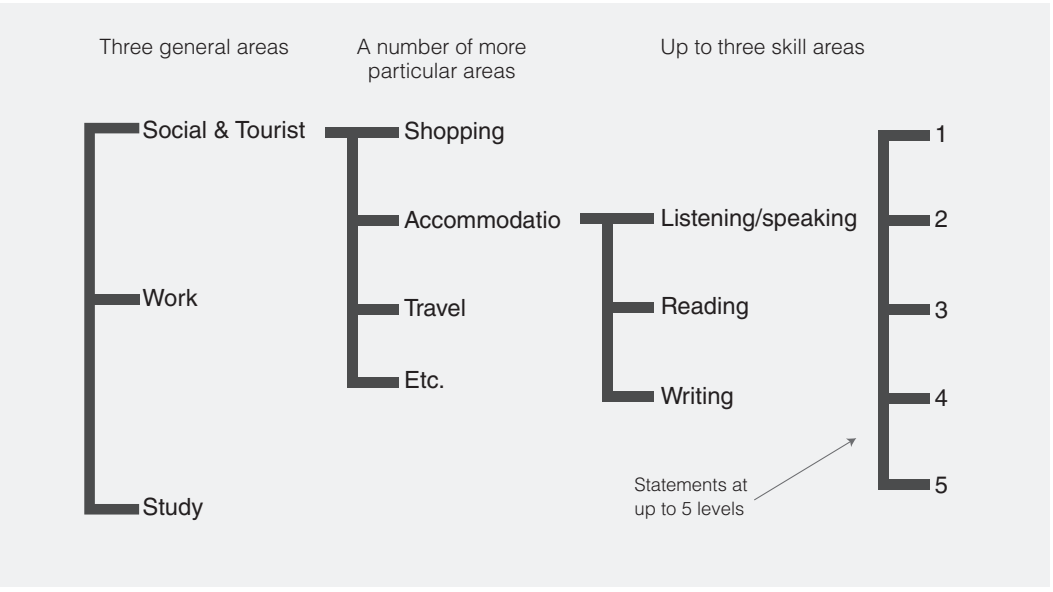

FIgURA 1. Estructura de las escalas del proyecto Can Do de ALTE

El nivel A1 (MCER) se encuentra en pleno proceso de definición por el proyecto Can Do y equivaldría a un nivel 0 (inicial o nivel de acceso), mientras que los restantes se corresponden con los niveles A2 (nivel 1), B1 (nivel 2), B2 (nivel 3), C1 (nivel 4) y C2 (nivel 5) del MCER. Lo que realmente interesa para el análisis de este estudio es saber en qué lugar queda la competencia léxica y las

7 Alemán, catalán, danés, español, finés, francés, holandés, inglés, italiano, noruego, portugués y sueco. 
áreas relacionadas con el léxico de la alimentación. En este sentido, no existe una especificación clara ni una propuesta concreta de selección léxica, sino que solamente se expone una serie de parámetros que ayudan a separar los umbrales de nivel por materia.

Como se puede observar, en ninguno de los dos documentos (PCIC y MCER) se alude a criterios de selección léxica, sino que el proyecto se concibe como una pauta necesaria que complementa, a modo de indicaciones contextuales, la información de cada nivel sobre la capacidad del alumno para desenvolverse en determinadas situaciones, ayudando así a establecer diferencias entre los distintos umbrales. Los datos que ofrecen MCER y PCIC son generales, sin una aplicación expresa para cada uno de los umbrales, ya que, a pesar de reflejar una voluntad de caracterización, el resultado de todo el proceso parece asociarse con una serie de condiciones extralingüísticas que no permiten fundamentar las ulteriores selecciones. Según estos resultados, queda de manifiesto que los manuales de ELE no tienen una fuente de referencia incuestionable.

A continuación, en el Cuadro 2, se indica la propuesta léxica de la alimentación que ofrece el PCIC, a partir de la cual se ha desarrollado el estudio contrastivo del léxico del nivel A1 que presentamos en $\S 3$.

CuADro 2. Selección léxica de la alimentación del Pcic

\begin{tabular}{|c|c|c|c|c|c|c|}
\hline \multicolumn{2}{|c|}{ DIETA Y NUTRICIÓN } & \multicolumn{2}{|c|}{ BEBIDA } & \multirow{2}{*}{$\begin{array}{c}\text { ALIMENTOS } \\
\text { Carne }\end{array}$} & \multirow{2}{*}{$\begin{array}{l}\text { Platos } \\
\text { Sopa }\end{array}$} & \multirow{2}{*}{$\begin{array}{c}\text { RESTAURANTE } \\
\text { Bar }\end{array}$} \\
\hline Desayuno & Desayunar & Agua (mineral) & & & & \\
\hline & & Leche & & Pescado & Ensalada & Restaurante \\
\hline \multirow[t]{2}{*}{ Comida } & Comer & Té & Beber & Verdura & Paella & Camarero \\
\hline & & $\begin{array}{c}\text { Café (solo, } \\
\text { con leche, } \\
\text { cortado) }\end{array}$ & & Fruta & Tortilla & Mesa \\
\hline Merienda & Merendar & Cerveza & & Leche & $\begin{array}{l}\text { Primer/se- } \\
\text { gundo plato }\end{array}$ & El menú \\
\hline \multirow[t]{5}{*}{ Cena } & Cenar & Vino & & Huevos & Postre & La cuenta \\
\hline & & & & Pan & & \\
\hline & & & & Bocadillo & & \\
\hline & & & & Sándwich & & \\
\hline & & & & Hamburguesa & & \\
\hline
\end{tabular}


Como puede observarse, se hallan 42 palabras que sientan las bases del aprendizaje del vocabulario de la alimentación en español. Estas se subdividen en cinco apartados: "dieta y nutrición", "bebida", "alimentos", "platos" y "restaurante".

\section{El léxico de la alimentación en los manuales de ELE}

En esta parte presentamos el léxico de la alimentación correspondiente al nivel A1 en los ocho manuales de ELE. Para ello, lo hemos dividido en cinco apartados: en $\$ 3.1$, presentamos la caracterización sintética de los manuales y su clasificación cronológica a partir de su fecha de publicación; en $\$ 3.2$, los datos de frecuencias y disponibilidad léxica de las 42 palabras de la propuesta léxica del PCIC; en §3.3, revisamos las nociones específicas del PCIC del ámbito de la alimentación que aparecen en los manuales estudiados; en \$3.4, comentamos qué palabras aparecen en los manuales del nivel A1 y que no se contemplan en las nociones específicas del PCIC, y, por último, en $\S 3.5$, recapitulamos los resultados obtenidos de la comparación entre las nociones específicas del PCIC y el léxico de los manuales estudiados.

Dadas las magnitudes de un análisis de todo el material a nuestro alcance, hemos seleccionado una muestra representativa de ocho manuales, escogidos en función de su uso y diversidad y con el propósito de contar con una visión general del trabajo editorial en este ámbito: manuales de las editoriales Difusión (Gente 1, Aula 1: curso de español y Bitácora 1), Edinumen (Nuevo Prisma A1: curso de español para extranjeros), Anaya (Sueña 1), Edelsa (Preparación al DELE A1), SM (Nuevo ELE inicial 1: curso de español para extranjeros) y Sociedad General Española de Librería (SGEL) (Vocabulario ELE B1: léxico fundamental de español de los niveles Al a B1). Esta selección nos permite analizar manuales de distinta naturaleza (manuales de enseñanza, de preparación de exámenes y centrados en el aprendizaje de vocabulario), de modo que así contamos con una visión integradora de los distintos tipos de obras existentes para el aprendizaje del vocabulario de la alimentación en el nivel A1. 


\subsection{Caracterización de los manuales estudiados}

Los manuales estudiados se han seleccionado teniendo en cuenta su diversidad en cuanto a metodología de enseñanza. Los hemos agrupado en tres periodos: 20012004, 2009-2011 y 2013-2014. Dentro del periodo 2001-2004, incluimos los manuales Sueña 1 (2001), Aula 1 (2003) y Gente 1 (2004). El periodo 2009-2011 está compuesto por Preparación al DELE Al (2009), Nuevo ELE inicial 1 (2010) y Bitácora 1 (2011). Y el periodo 2013-2014 por Nuevo Prisma Nivel Al (2013) y Vocabulario ELE B1 (2014). El hecho de que tres de los manuales estudiados aparezcan antes de 2004 nos permitirá saber si la publicación del PCIC en 2006 influyó significativamente en su selección léxica.

En nuestro análisis de los manuales no ha sido posible identificar referencias a los criterios seguidos para seleccionar el léxico que incluyen en sus actividades. A este respecto, hemos seleccionado los manuales más importantes de cada tipo para asegurarnos de que nuestra hipótesis no se circunscribe a un tipo de manual, sino que lo que se afirma en ella es una tendencia generalizada.

\subsection{Datos de frecuencias y de disponibilidad léxica}

Los datos relativos a las frecuencias de las 42 palabras objeto de análisis nos ayudan a contar con más información acerca de su posicionamiento en el uso de la lengua. En el CREA, destacamos que hay 5 voces que se hallan entre las 1000 más frecuentes — agua (207), carne (923), cuenta (174), mesa (611) y vino (766) — y 14 palabras entre las 1000 y las 5000 siguientes — bar (2723), beber (3318), café (1117), cena (2508), cerveza (4208), comer (1317), comida (1426), desayuno (4814), huevos (2572), leche (1239), pan (1177), pescado (3049), restaurante (3435) y té (4055). Del resto de palabras, hay 20 que se hallan a partir de la posición 5000 y 3 que no aparecen — café con leche, café cortado y café solo. Las 5 palabras de nuestro elenco que se hallan entre las 1000 primeras palabras del CREA suelen estar presentes en los manuales estudiados, si bien agua falta en Sueña 1; carne, en Sueña 1 y en Nuevo ELE inicial 1; cuenta, en Sueña 1 y en Vocabulario ELE B1; mesa, en Aula y vino, en Nuevo Prisma Nivel A1. De 14 palabras que se hallan entre las 1000 y las 5000 más frecuentes del CREA, 
solo restaurante se encuentra en todos los manuales. Bar (Preparación al DELE A1), beber (Nuevo Prisma 1), cerveza (Nuevo Prisma 1), comida (Sueña 1) y huevos (Nuevo ELE inicial) se obvian en un manual cada uno (el indicado entre paréntesis), cena, comer, desayuno, leche y pescado, en dos (Sueña 1 y Nuevo ELE inicial 1) y solo pan, en tres (Aula 1, Nuevo Prisma 1 y Nuevo ELE inicial 1). Por lo tanto, a la luz de estos datos, podemos afirmar que, en líneas generales, las palabras recogidas en los manuales se corresponden con las palabras frecuentes, pero observamos ciertas ausencias que resulta difícil explicar si no es por razones de utilidad o de otro tipo, pero que en cualquier caso desconocemos.

Los datos referentes a la disponibilidad léxica de las 42 palabras objeto de estudio son cuantitativamente inferiores con respecto a los ofrecidos por el CREA, puesto que, aunque en el ámbito de la alimentación solo contamos con datos relativos a Valencia y Soria, son datos bastante coincidentes. En el listado de Valencia se encuentran 22 voces (52\%) y en el de Soria, 24 (57\%). El grado de coincidencia entre ambos listados es alto, pues los dos recogen 21 de las voces totales. El de Valencia solo incorpora una palabra ausente en el listado de Soria —agua mineral - y el de Soria 3, con respecto al de Valencia — menú, primer plato y segundo plato. La coincidencia entre ambos listados también se halla, en líneas generales, en las posiciones en las que se ordenan las palabras recogidas, como se indica en el Cuadro 3.

Cuadro 3. Posiciones de las 42 palabras en listados de disponibilidad léxica

\begin{tabular}{|c|c|c|c|c|c|}
\hline PCIC & Dispolex Valencia & Dispolex Soria & PCIC & Dispolex Valencia & Dispolex SoriA \\
\hline 1. Agua & 1 & 1 & 12. Cena & & \\
\hline 2. Agua mineral & 302 & & 13. Cenar & & \\
\hline 3. Bar & & & 14. Cerveza & 17 & 9 \\
\hline 4. Beber & & & 15. Comer & & \\
\hline 5. Bocadillo & 285 & 117 & 16. Comida & & \\
\hline 6. Café & 59 & 49 & 17. Desayunar & & \\
\hline 7. Café con leche & & & 18. Desayuno & & \\
\hline 8. Café cortado & & & 19. El menú & & 329 \\
\hline 9. Café solo & & & 20. Ensalada & 102 & 127 \\
\hline 10. Camarero & & & 21. Fruta & 77 & 35 \\
\hline 11. Carne & 16 & 20 & 22. Hamburguesa & 51 & 74 \\
\hline
\end{tabular}


Hacia una propuesta de selección léxica en manuales de español como lengua extranjera de nivel A1 79

CuAdro 3. Posiciones de las 42 palabras en listados de disponibilidad léxica (continuación)

\begin{tabular}{|c|c|c|c|c|c|}
\hline PCIC & Dispolex Valencia & DispoleX SoriA & PCIC & Dispolex $V_{\text {ALENCIA }}$ & DISPOLEX SoriA \\
\hline 23. Huevos & 24 & 50 & 34. Primer plato & & 304 \\
\hline 24. La cuenta & & & 35. Restaurante & & \\
\hline 25. Leche & 7 & 15 & 36. Sándwich & 156 & 137 \\
\hline 26. Leche* & 7 & 15 & 37. Segundo plato & & 308 \\
\hline 27. Merendar & & & 38. Sopa & 79 & 56 \\
\hline 28. Merienda & & & 39. Té & 99 & 102 \\
\hline 29. Mesa & & & 40. Tortilla & 123 & 122 \\
\hline 30. Paella & 56 & 182 & 41. Verdura & 68 & 37 \\
\hline 31. Pan & 15 & 6 & 42. Vino & 2 & 3 \\
\hline 32. Pescado & 23 & 16 & & & \\
\hline 33. Postre & 310 & 268 & Total & $\begin{array}{c}22 \\
(52.38 \%)\end{array}$ & $\begin{array}{c}24 \\
(57.14 \%)\end{array}$ \\
\hline
\end{tabular}

* La palabra leche aparece repetida porque, en un caso, se considera como bebida y, en el otro, como lácteo/alimento.

Como se observa en el Cuadro 3, hay 20 términos del PCIC que no se incluyen en la lista de Valencia (47.61\%) y 18 términos que no aparecen en la de Soria (42.85\%). Por otra parte, cabe destacar que 7 de los 10 términos más disponibles en los datos de Dispolex Valencia no aparecen en el PCIC - tomate, lechuga, güisqui, coca cola, arroz, naranja y manzana — ni 6 de los 10 términos más disponibles de la lista de Dispolex Soria - coca cola, güisqui, lentejas, macarrón, zumo y tomate. Si bien no es un dato contundente, sí es significativo, sobre todo por la coincidencia entre ambos listados, pues entre estos 7 y 6 de los 10 términos más disponibles en el listado de Valencia y Soria, respectivamente, coinciden tres de ellos: tomate, güisqui y coca cola.

De estos 6 y 7 términos más disponibles en el listado de Valencia y Soria, respectivamente, solo coca cola, lentejas y macarrones pertenecen al nivel A1, de acuerdo con la clasificación del PCIC. El resto — arroz, lechuga, manzana, naranja, tomate y zumo - pertenece al nivel A2. La palabra güisqui no aparece en ningún manual y coca cola solo se incluye en Bitácora 1. Por lo tanto, observamos la falta de correspondencia entre los listados de disponibilidad léxica del ámbito de la alimentación que existen hasta ahora y el léxico que incluyen los manuales.

Por otra parte, hay términos que aparecen al final del listado y que se incluyen en la mayoría de los manuales, como por ejemplo café (presente en todos 
los manuales), verdura (presente en todos salvo en Nuevo ELE inicial 1) o postre (solo ausente en Nuevo ELE inicial 1). Hay otras voces que, en cambio, están ausentes en una gran mayoría de los manuales estudiados, como hamburguesa —ausente en 5 manuales: Sueña 1, Aula 1, Gente 1, Nuevo ElE inicial 1, Vocabulario ELE B1-y sándwich —ausente en 7 manuales: Sueña 1, Aula 1, Gente 1, Nuevo ele inicial 1, Bitácora 1, Nuevo Prisma Nivel A1, Vocabulario ELE B1.

\subsection{Las nociones específicas del PCIC en los manuales de ELE}

En este apartado se exponen los resultados del análisis de las nociones específicas del PCIC del ámbito de la alimentación que aparecen en los manuales estudia$\operatorname{dos}^{8}$ —en donde se realiza una propuesta del léxico fundamental del ámbito de la alimentación que divide en siete subapartados: "dieta y nutrición", "bebida", "alimentos", "recetas", "platos", "utensilios de cocina y mesa" y "restaurante" (Figura 2). Para la interpretación correcta de los datos, hay que tener presente que la fecha de publicación del PCic es 2004. Si bien no hemos contrastado estos datos con los de los corpus, porque excederíamos los límites planteados en este estudio, sí consideramos que es pertinente referirse a ellos con tal de que pueda observarse el grado de diversidad entre los manuales estudiados desde el punto de vista del léxico que usan para elaborar sus actividades.

Los manuales que menos términos del PCIC incorporan de los considerados de nivel A1 son Sueña 1 y Nuevo ELE inicial 1, con aproximadamente 50\%, diferenciándose notablemente del resto. También Nuevo Prisma Nivel Al presenta un porcentaje alto de omisión (40.5\%). Preparación al DELE Al es el manual que más cumple con lo expresado en el PCIC. Cabe señalar que los únicos de estos manuales que hacen alguna referencia al PCIC en cuanto a la organización de contenidos son Vocabulario ELE B1, Nuevo Prisma 1 y Preparación al DELE A1, pese a que los resultados, una vez analizado el léxico, no corresponden en todos los casos con estas indicaciones. Solamente tres de los ocho manuales analiza-

8 En el Apéndice 1 se recogen en una misma tabla las nociones específicas que aparecen en cada uno de los manuales estudiados (niveles A1 y A2).

9 En el nivel que nos ocupa en nuestro estudio (A1), los subapartados "utensilios de cocina y mesa" y "recetas" no son necesarios, ya que forman parte del nivel A2. 


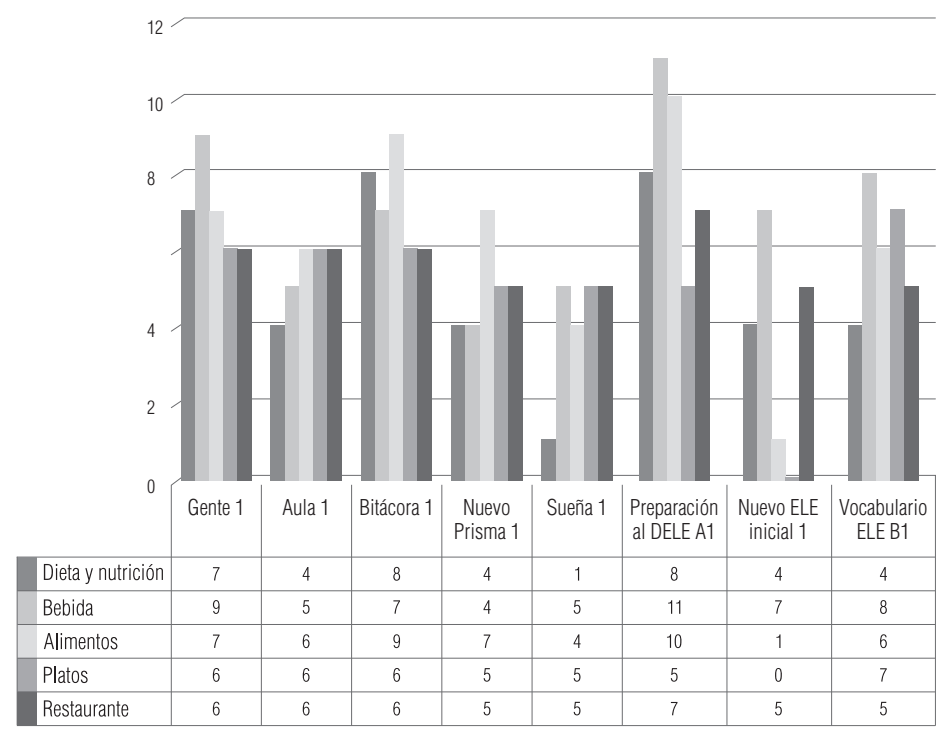

FIgURA 2. Nociones específicas del PCIC por temas en los manuales de ELE

dos (Preparación al DELE A1, Bitácora 1 y Gente 1) se aproximan a la propuesta recomendada por el documento de referencia oficial (PCIC). Entre los manuales restantes se comparte léxico de la propuesta del PCIC, a pesar de que su uso no se justifica, entre otras cosas, porque ni los propios documentos de referencia oficiales lo expresan con detalle.

\subsection{Palabras de los manuales de ELE (A1) que no se contemplan en las nociones específicas del PCIC (A1)}

En este apartado exponemos el resultado del análisis de las palabras de la alimentación que aparecen en los manuales del nivel A1 y que no se contemplan en las nociones específicas del PCIC en el nivel A1.

En la Figura 3 aparecen dos tipos de datos relativos a los ocho manuales estudiados: 1) voces que los manuales incorporan y no se recogen en el nivel A1 del PCIC; ${ }^{10}$ 2) palabras que pertenecen al nivel A2 del PCIC de las incorpo-

${ }^{10}$ En el Apéndice 2 se recogen en una misma tabla las palabras de los niveles A1 que no se con- 


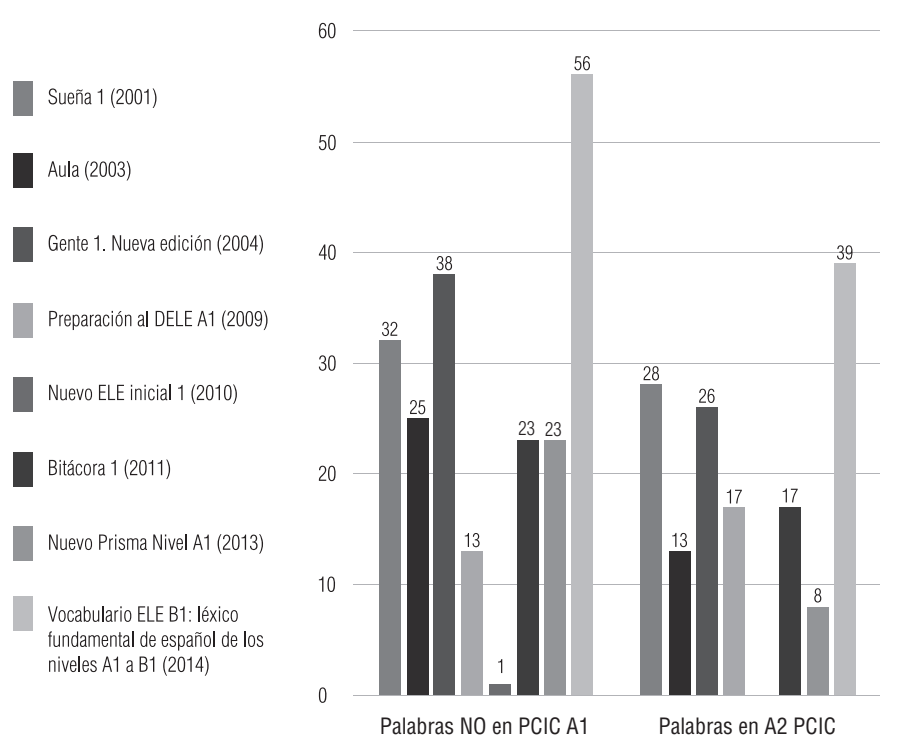

Figura 3. Palabras de los manuales de ElE (A1) que no se contemplan en las nociones específicas del PCIC (A1)

radas por los manuales para el nivel A1. Como se observa en el gráfico, el manual que más se aleja del PCIC es Vocabulario ELE B1, que incorpora 56 voces que no se contemplan en el nivel A1 del PCIC y 39 que el PCIC considera propias del nivel A2, curiosamente se trata del manual más actual de los analizados, 2014. En la misma línea encontramos los manuales Gente 1, con 38 y 26 palabras, respectivamente, y Sueña 1, con 32 y 28, respectivamente. Los manuales Aula 1, con 25 y 13, Preparación al DELE A1, con 13 y 17, y Bitácora 1, con 23 y 17.

\subsection{Resultados de la comparación}

De acuerdo con los datos recopilados, el léxico de la alimentación del nivel A1 en los manuales no presenta demasiadas modificaciones en comparación con la propuesta del PCIC, salvo algunas excepciones. Del total de 42 ítems ofrecidos

\footnotetext{
templan en las nociones específicas del PCIC.
} 
por el PCIC, los manuales que muestran más deficiencias con respecto al léxico básico que contempla el documento de referencia del Instituto Cervantes son Nuevo ELE inicial 1 y Sueña 1, con tan solo 17 (40.4\%) y 20 (47.6\%) palabras, respectivamente. De estos dos manuales, Nuevo ELE inicial 1 es el único que no tiene ninguna palabra del subtema "Platos", de modo que sus carencias, más allá del ámbito cualitativo, son notables en el cuantitativo al prescindir de una categoría básica perteneciente al ámbito de la alimentación. En la Figura 4 se ofrece el ranking de los manuales según se aproximen a la cifra recomendada por el PCIC, de mayor a menor coincidencia.

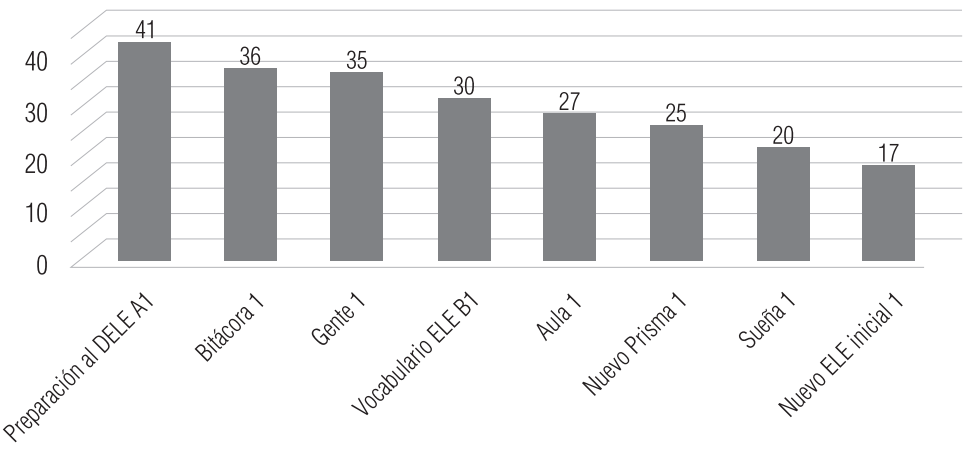

FIGURA 4. Ranking de los ocho manuales ELE según su coincidencia con el léxico de la alimentación propuesto por el PCIC

A partir de estos resultados, de los manuales Vocabulario ELE B1, Nuevo Prisma 1 y Preparación al DELE A1, que son los únicos que declaran seguir el PCIC $^{11}$ por lo que respecta a la selección léxica realizada, Preparación al DELE Al se constituye como el manual que contiene la mayoría del léxico del PCIC, a excepción de solamente una palabra, mientras que Vocabulario ELE B1 con 30 (de 42) y Nuevo Prisma 1 con 25 (de 42) se mantienen bastante por debajo. En definitiva, aunque estos manuales sí hacían referencia al PCIC, lo hacían en cuanto a la clasificación de grupos, lo que puede entenderse aquí como los diferentes subtemas o subclasificaciones del léxico de la alimentación y, por consi-

${ }^{11}$ Preparación al DELE Al no lo indica explícitamente, pero es fácilmente identificable, ya que se constituye a partir de modelos de examen del DELE (Instituto Cervantes). 
guiente, debían tener conocimiento de la propuesta léxica del PCIC. No obstante, la selección de los dos últimos casos no es del todo eficiente, tratándose, además, de dos manuales muy recientes (Nuevo Prisma 1, 2013, y Vocabulario ELE $B 1,2014)$.

Entre todas estas propuestas que engloban la realidad de las selecciones léxicas de manuales de ELE en España y a partir de la consideración de las exclusiones en cada caso, el resultado de los análisis demuestra que ninguno de los manuales logra recopilar todo el léxico indicado en el PCiC, aunque Bitácora 1 y Preparación al DELE Al siguen muy de cerca su propuesta. El primero no incorpora en su repertorio de la alimentación las palabras paella y sándwich, mientras que el manual destinado a la preparación del examen DELE omite bar. En el caso de Bitácora 1, teniendo en cuenta que se trata de un manual transgresor y original, podemos intuir que la falta del vocablo paella se puede deber a una reacción contra el fomento de los tópicos alimenticios de España, ya que el hecho de considerar paella como una palabra del nivel básico no solamente rompe con el espíritu panhispánico de ir más allá de la realidad española, sino que además ocupa el lugar 18767 del corpus CREA y, por consiguiente, no es una palabra que conste de una frecuencia lo suficientemente alta como para ser considerada fundamental. No obstante, es preciso recordar que el PCIC advierte que "las series de exponentes responden a un criterio de selección basado en la variedad centro-norte peninsular española y en las características idiosincrásicas de España" (PCIC, "Nociones Específicas. Introducción"), de manera que no se puede exigir el reflejo de otras realidades de la geografía hispanohablante, a pesar de que, como es lógico, debería existir una voluntad de ofrecer un léxico básico panhispánico.

Para poder reflexionar sobre una posible selección léxica de la alimentación en el nivel A1, es interesante, en un primer momento, observar las coincidencias, esta vez en términos de ausencia de léxico desde un punto de vista cuantitativo y cualitativo, según el caso. En la Figura 5 se reflejan las ausencias de léxico más destacadas con el objetivo de discernir sobre las causas de esta ausencia al tratarse de una falta análoga en los distintos manuales analizados.

Tal y como se hace patente en la Figura 5, las principales ausencias (desde un punto de vista cuantitativo) tienen lugar en los ámbitos de "Alimentos" y "Dieta y nutrición". En el caso de "Dieta y nutrición", la falta se produce en todos los verbos — beber, merendar, cenar, comer y desayunar-, una ausencia 


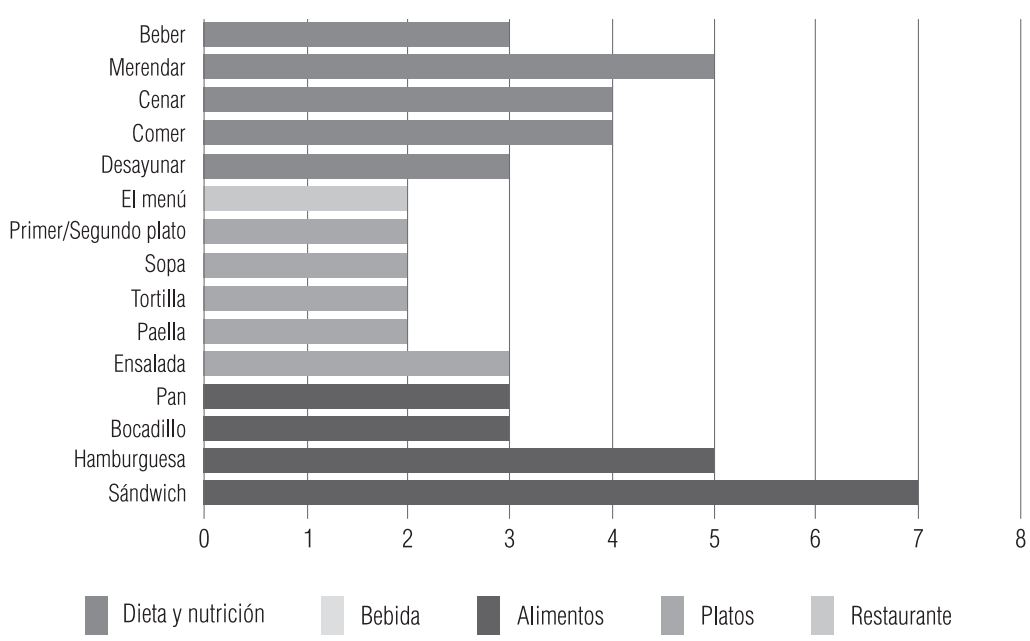

Figura 5. Ausencias destacadas del léxico de la alimentación recomendado por el PCiC para el nivel A1 en los manuales ELE

compartida por prácticamente un tercio de los manuales de ELE. Esto puede deberse a la productividad de este tipo de léxico, puesto que palabras como desayuno, comida o cena representan actividades desde un punto de vista semántico y, por ello, en su definición se halla la misma acción de desayunar, comer o cenar. En los casos de manuales elaborados por medio del enfoque comunicativo, este proceder puede ser recurrente. En cambio, merendar solo se incorpora en los manuales Gente 1, Bitácora 1 y Preparación al DELE A1, manuales que, curiosamente, incluyen más léxico en sus selecciones. Además, dicho verbo se encuentra en la posición 32604 del CREA, solamente por delante de hamburguesa y sándwich, que son las palabras que más ausencias presentan del conjunto de las analizadas, de manera que la frecuencia es un factor decisivo en este caso.

Por lo que respecta a hamburguesa, dicha omisión afecta a los manuales Gente 1, Sueña 1 y Vocabulario ELE B1. En este caso, esa palabra forma parte, al igual que sándwich, de las últimas posiciones del índice de frecuencia del CREA (37 633), por detrás de las 20000 palabras más frecuentes del español según el corpus, y coincide que ambas son anglicismos. Todo apunta a que se ha tratado de una decisión personal de los creadores y no a un descuido, como sí lo parece la falta de bocadillo en Vocabulario ELE B1 y Sueña 1, al que se le suma la falta 
de carne, pescado y fruta. El manual de la editorial SM, Nuevo ELE inicial 1, no muestra ninguna de las palabras a excepción de leche, hecho totalmente injustificado pero que no nos sorprende por la organización poco sistemática de este manual a la hora de presentar los diferentes centros de interés.

En cuanto a la palabra sándwich, se ofrece una serie de problemas que van más allá de su presencia o ausencia en los manuales de ELE. En primer lugar, a excepción de Preparación al DELE A1, que cumple con las diez formas presentadas en el PCIC, los siete manuales restantes rehúsan utilizar dicho vocablo. Se desconoce cuál ha sido la motivación que ha llevado a considerar sándwich como una de las formas básicas del nivel A1 en español en dicho manual, teniendo en cuenta que la lista de frecuencias del corpus CREA la sitúa en el último lugar de todas las propuestas por el PCIC, concretamente en el puesto 67 894, muy alejado de su predecesora hamburguesa en el puesto 37 633. El anglicismo tiene su origen en la gastronomía inglesa ${ }^{12} \mathrm{y}$, según el Diccionario panhispánico de dudas (DPD) de la RAE, se refiere al "conjunto de dos o más rebanadas de pan, normalmente de molde, entre las que se ponen distintos alimentos" (RAE, 2005). Por otra parte, existen otros indicios que hacen que se puedan tener dudas sobre el uso de la palabra sándwich en el nivel A1. Por una parte, en la definición del Diccionario de la lengua española (DRAE) de la Real Academia Española esta palabra se define como "Emparedado hecho con dos rebanadas de pan de molde entre las que se coloca jamón, queso, embutido, vegetales u otros alimentos", de cuya definición se puede extraer que el vocablo sándwich parece ser un tipo de emparedado, ya que la definición de emparedado del DRAE señala que se trata de una "Porción pequeña de jamón u otra vianda, entre dos rebanadas de pan de molde". De este modo, no se establece desde el punto de vista de la microestructura ningún tipo de relación sinonímica, ya que en ambos casos no hallamos remisión alguna, como sería habitual en los casos de sinonimia. Aun así, según el DPD, emparedado es el equivalente de sándwich en español y fue puesto en circulación a partir del siglo xIx; además, indica que su uso es preferible al del anglicismo sándwich, de manera que este diccionario sí contempla una relación de sinonimia entre ambos vocablos. Las propias incongruencias entre las obras de la RAE,

${ }^{12}$ Concretamente en el IV conde de Sandwich, John Montagu (1718-1792), de quien se cuenta que se alimentó de esta clase de comida para no abandonar una partida de cartas. 
aunque sabemos que solo el DRAE es el diccionario del español con valor estrictamente normativo, dan como resultado una situación resbaladiza en la que no queda del todo clara la relación semántica entre estas dos palabras, puesto que mientras la definición del DRAE alude a una relación de hiperonimia en la que emparedado sería hiperónimo de sándwich, el DPD parece catalogar dicha relación de sinonimia al rehusar el uso del anglicismo por ser innecesario en español. Otros diccionarios como, por ejemplo, Clave (Maldonado González, 1996) y el Diccionario de sinónimos y antónimos, de la editorial SM (Maldonado González, 1999), sí establecen una relación de sinonimia entre emparedado y sándwich, mientras que el Diccionario Salamanca sitúa como hiperónimo bocadillo, para identificar sándwich y emparedado como tipos de bocadillo 'con pan de molde'. El hecho de que un buen número de manuales decidan prescindir directamente de ese término y no hayan apostado por la variante española emparedado (puesto 63959 del CREA) podría deberse a la ambigüedad propiciada por la definición lexicográfica y las recomendaciones del DPD, que no son resolutivas en absoluto — aunque no contamos con evidencias de ello.

Problemáticas como las observadas no son exclusivas de los manuales que hemos seleccionado para esta investigación. Benítez y Zebrowski (1993) analizaron los cuatro manuales polacos de español más utilizados en ese país y encontraron que en uno de ellos, dirigido a principiantes, aparecía la palabra perdiz pero no la voz pájaro. A este respecto parece recomendable trabajar primero con las formas genéricas y pasar después a las más concretas. Un año después se publicó el trabajo de Benítez (1994) en el que comparó el léxico disponible de los 16 ámbitos clásicos de 257 estudiantes del Curso de Orientación Universitaria (COU), ${ }^{13}$ lo que dio lugar a 2340 palabras — total de lexemas computados — reducidas a 107 vocablos — los diferentes_,${ }^{14}$ con varios manuales de la época, y llegó a la conclusión de que los manuales usaban muchas voces con un alto índice

${ }^{13}$ De acuerdo con el sistema educativo en España diseñado en la Ley General de Educación de 1970, el cou era una enseñanza no obligatoria que constaba de un solo curso, equivalente al último curso de bachillerato de la Ley Orgánica General del Sistema Educativo (LOGSE) de 1990, y que se cursó por última vez durante el año académico 1998-1999.

${ }^{14}$ En disponibilidad se entiende por palabra cada uno de los lexemas computados y, por vocablo, solo los diferentes. 
de disponibilidad pero también incluían algunas de bajo índice o incluso de índice cero. En ellos se hallan voces que solo aparecen una vez en todo el manual.

\section{Hacia una propuesta de selección del léxico de la alimentación del nivel $A 1$}

A partir de la consideración de estos datos y del criterio de la limitación objetiva corregida, se puede establecer una selección del léxico básico de la alimentación en el nivel A1 que contemple, a su vez, los datos extraídos - convergencias y divergencias- de los diferentes manuales de ELE (véase Cuadro 4).

Cuadro 4. Propuesta de selección léxica de la alimentación para el nivel A1

\begin{tabular}{|c|c|c|c|c|c|c|}
\hline \multicolumn{2}{|c|}{ DIETA Y NUTRICIÓN } & \multicolumn{2}{|c|}{ BEBIDA } & \multirow{2}{*}{$\begin{array}{l}\text { Alimentos } \\
\text { Carne }\end{array}$} & \multirow{2}{*}{$\begin{array}{l}\text { PLATOS } \\
\text { Sopa }\end{array}$} & \multirow{2}{*}{$\begin{array}{c}\text { RESTAURANTE } \\
\text { Bar }\end{array}$} \\
\hline Desayuno & Desayunar & Agua & Beber & & & \\
\hline Comida & Comer & Leche & & Pescado & Ensalada & Restaurante \\
\hline Merienda & Merendar & Té & & Verdura & Paella & Camarero \\
\hline \multirow[t]{7}{*}{ Cena } & Cenar & $\begin{array}{c}\text { Café (solo, } \\
\text { con leche, } \\
\text { cortado) }\end{array}$ & & Fruta & Tortilla & Mesa \\
\hline & & Cerveza & & Leche & $\begin{array}{l}\text { Primer/se- } \\
\text { gundo plato }\end{array}$ & El menú \\
\hline & & Vino & & Huevos & Postre & La cuenta \\
\hline & & & & Pan & & \\
\hline & & & & Bocadillo & $\longrightarrow$ & \\
\hline & & & & Sándwich & & \\
\hline & & & & Hamburguese & & \\
\hline
\end{tabular}

Entre los datos incluidos en el Cuadro 4, no recogemos los relativos a la disponibilidad, por no ser tan concluyentes como los de frecuencias. En este sentido, en el caso de que en un futuro contáramos con más datos de disponibilidad léxica del ámbito de la alimentación que corroboraran los actuales, deberíamos plantear la inclusión de los tres términos más disponibles que coinciden entre el listado de Valencia y el de Soria: tomate, güisqui y coca cola.

Según los datos que se han ido ofreciendo, una selección léxica de la alimentación debería prescindir de sándwich y hamburguesa porque no pueden jus- 
tificarse en términos de frecuencia, ya que se hallan demasiado alejadas de las demás palabras y no hay ninguna razón que justifique su pertenencia al léxico básico del español en el nivel A1, a menos que se decida escogerlas por su utilidad. Además, estas voces incumplen la regla de la eficacia, puesto que se trata de dos palabras de aplicación muy concreta, y carecen de productividad. En cuanto a bocadillo, que se encuentra en el límite de las 25000 palabras más frecuentes del CREA —-posición 24 356-, resulta útil por dos razones: en primer lugar, porque es un alimento típico de la gastronomía española — aunque se trate de una apreciación extralinguiística- $-y$, en segundo lugar, porque es una palabra eficaz en varios contextos, ya que podemos referirnos a bocadillo como hiperónimo de un grupo y así referirnos a bocadillo de jamón, por ejemplo. A su vez, bocadillo pasaría a formar parte del grupo "Platos", ya que no es un alimento propiamente dicho. A su vez, apostamos por mantener los verbos - cenar, comer, desayunar y merendar-, a pesar de su productividad, ya que el estudiante extranjero no tiene por qué tener ese conocimiento implícito de la lengua y, además, estos forman parte del significado de las palabras desayuno, comida, merienda y cena, de manera que son fundamentales para la comprensión de dichos vocablos en un nivel inicial.

Asimismo, esta propuesta deriva de los datos que se han obtenido de los manuales de ELE que no incorporan la palabra sándwich, con la excepción de Preparación al DELE A1. Por lo que respecta a hamburguesa, dentro de los cinco manuales que no la incorporan están Gente 1, Vocabulario ELE B1 y Aula 1, buenas referencias para la selección del léxico según los datos obtenidos. De este modo, con esta propuesta pretendemos, por una parte, dejar constancia de la realidad reflejada por los hacedores de manuales de ELE en España y justificar las ausencias correspondientes en cada caso y, por otra, pretendemos demostrar que en el proceso de selección objetiva intervienen muchas variantes que no siempre coinciden con lo propuesto en los documentos de referencia, como, en este artículo, el PCIC.

El análisis de la selección léxica de cada manual nos ha permitido obtener una visión precisa tanto a nivel cualitativo como cuantitativo del léxico ofrecido por los manuales de ELE. Las carencias importantes de léxico pueden justificarse por los motivos a los que hemos hecho referencia en términos de frecuencia y productividad, aunque en muchos casos podría ser que se deban a descuidos por 
parte de los autores de materiales o bien a una selección del léxico estrictamente subjetiva o bien basada en un criterio fundamentado en la experiencia profesional, que, como tal, sería perfectamente válido si así se explicitara, a pesar de que es difícil de sostener con datos objetivos.

\section{Conclusiones}

Los resultados de la observación y análisis de los datos en relación con los objetivos y la hipótesis del estudio nos permiten establecer las conclusiones siguientes.

En primer lugar, ha quedado confirmada nuestra hipótesis según la cual en los manuales españoles actuales de ELE no se especifican los criterios que se siguen para seleccionar el léxico que incluyen en sus actividades ni se tienen en cuenta los datos de frecuencias y de disponibilidad proporcionados por los diversos corpus hoy existentes.

Por otro lado, se ha demostrado que en ningún manual se especifica si se sigue o no algún criterio en la selección del léxico. Es más, ni siquiera los propios documentos oficiales de referencia explican cuáles son los criterios que han seguido para la selección del léxico. El MCER, como es lógico, aporta una información funcional y se limita a sugerir cómo llevar a cabo la selección del léxico, donde las situaciones comunicativas juegan un papel importante. El PCIC, a pesar de haberse guiado por los objetivos generales para los niveles de referencia del español y del proyecto Can Do de ALTE, no facilita criterios de selección léxica, sino únicamente pautas de comportamiento lingüístico del alumno en situaciones sociales. La selección léxica del PCiC representa hoy en día el único modelo de referencia para los creadores de manuales y demuestra las analogías que hay entre las selecciones de los distintos manuales de ELE, a pesar de que no se puede considerar una selección léxica objetiva corregida teniendo en cuenta que no se explica ni se justifica a partir de las referencias a las que nos remite (objetivos generales y proyecto Can Do de ALTE).

Asimismo, la información analizada ha demostrado, tal y como se afirmaba en nuestra hipótesis, que los datos de frecuencias y de disponibilidad no son considerados por los hacedores de manuales. El contraste entre las voces de los manuales y los índices de frecuencia y de disponibilidad léxicas nos permite afirmar que existe falta de correspondencia entre ambos. La frecuencia ha teni- 
do un papel importante en el proceso de elaboración de los manuales, aunque también ha servido para constatar que el léxico frecuente no es siempre el léxico fundamental, sino que una buena parte del léxico necesita activarse en función del contexto y de la situación y, por tanto, hay que analizarlo en términos de productividad y eficacia o utilidad. La comparación de las palabras analizadas con los dos índices de disponibilidad léxica de la alimentación a nuestra disposición nos ha permitido observar la falta de correspondencia existente entre los listados de disponibilidad léxica del ámbito de la alimentación y el léxico que incluyen los manuales. La selección léxica de la alimentación de los manuales refleja la variabilidad y falta de criterios objetivos para llevar a cabo esta labor, puesto que no existen criterios homogéneos entre manuales, sobre todo partiendo de las divergencias existentes entre las distintas selecciones analizadas. El léxico de la alimentación más recurrente entre manuales coincide con la propuesta del PCIC, cuyas diferencias principales se encuentran en la falta de léxico básico y no en la incorporación de nuevas palabras. La ausencia de léxico básico en los manuales se debe a causas de distinta índole, como la selección subjetiva, la falta de coherencia interna de los manuales y la inexistencia de un vocabulario fundamental del español. Además, no existe ningún dato que demuestre que la tipología diferenciada de los manuales haya influenciado en las selecciones léxicas que se han llevado a cabo. El problema de esta carencia abre las puertas a la reflexión sobre cuál debería ser el léxico fundamental del español, un campo de estudio en el que aún queda mucho por explorar. Las aportaciones de diferentes tipos de especialistas del ámbito de la lingüística aplicada a la enseñanza de lenguas extranjeras serán fundamentales para que se puedan producir resultados lingüísticos útiles en la elaboración de materiales didácticos que conduzcan a que cualquier estudiante de ELE pueda adquirir de la manera más fiable y rápida posible el léxico fundamental del idioma.

\section{Referencias}

Alonso Pérez-Ávila, Elena (2007). El corpus lingüístico en la didáctica del léxico del español como LE. Boletín de la Asociación para la Enseñanza del Español como Lengua Extranjera, $37,11-32$. 
Association of Language Testers in Europe (alte) (2002). The alte Can Do Project. Recuperado de https://www.cambridgeenglish.org/Images/28906-alte-can-do-document.pdf

Alvar Ezquerra, Manuel (2005). La frecuencia léxica y su utilidad en la enseñanza del español como lengua extranjera. En María Auxiliadora Castillo Carballo (Coord.), Las gramáticas y los diccionarios en la enseñanza del español como segunda lengua: deseo y realidad. Actas del XV Congreso Internacional de la Asele (pp. 19-39). Sevilla: Universidad de Sevilla.

Álvarez Martínez, María Ángeles (2001). Sueña 1. Madrid: Anaya

Ávila Martín, María del Carmen (2010). Estadística y lingüística de corpus: implicaciones pedagógicas en la enseñanza y el aprendizaje del léxico. Cauce. Revista internacional de Filología, Comunicación y sus Didácticas, 33, 163-175.

Baralo, Marta (2007). Adquisición de palabras: redes semánticas y léxicas. En Carmen Pastor Villalba (Coord.), Actas del Programa de formación para el profesorado de español como lengua extranjera del Instituto Cervantes de Múnich (2006-2007) (pp. 384-399). Múnich: Instituto Cervantes.

Bartol Hernández, José Antonio (2006). La disponibilidad léxica. Revista Española de Lingüística, 36, 379-391.

Bartol Hernández, José Antonio (2010). Disponibilidad léxica y selección del vocabulario. En Rosa María Castañer Martín \& Vicente Lagüéns Gracia (Eds.), De moneda nunca usada: estudios filológicos dedicados a José M. Enguita Utrilla (pp. 85-107). Zaragoza: Institución Fernando el Católico.

Benítez Pérez, Pedro (1994). Léxico real/léxico irreal en los manuales de español para extranjeros. En Salvador Montesa Peydró \& Antonio Garrido Moraga (Coords.), Actas del II Congreso Nacional de Asele. Español para extranjeros: didáctica e investigación (pp. 325-334). Málaga: Asociación para la Enseñanza del Español como Lengua Extranjera.

Benítez Pérez, Pedro (2009). ¿Qué vocabulario hay que enseñar en las clases de español como lengua extranjera? Monográficos marcoELE, 8, 9-12.

Benítez Pérez, Pedro, \& Zebrowski, Jerzy (1993). El léxico español en los manuales polacos. En Salvador Montesa Peydró \& Antonio Garrido Moraga (Coords.), Actas del Tercer Congreso Nacional de Asele. El español como lengua extranjera: de la teoría al aula (pp. 223-230). Málaga: Asociación para la Enseñanza del Español como Lengua Extranjera.

Bergenholtz, Henning (2003). User-oriented understanding of descriptive, proscriptive and prescriptive lexicography. Lexikos, (13), 65-80.

BogaARds, PAul (1994). Le vocabulaire dans l'apprentissage des langues étrangères. París: Hatier Didier. 
Borobio Carrera, Virgilio (2010). Nuevo ele inicial 1. Curso de español para extranjeros: libro del alumno. Madrid: SM.

Cerdeira Núñez, Paula, \& Ianni José Vicente (2013). Nuevo Prisma Nivel A1. Curso de español para extranjeros. Madrid: Edinumen.

Conejo, Emilia, Chamorro, María Dolores, \& Pablo, Martínez Gila (2011). Bitácora 1. Barcelona: Difusión.

Consejo de Europa (2002). Marco Común Europeo de Referencia para las Lenguas: aprendizaje, enseñanza, evaluación. Madrid: Secretaría General Técnica del MEC/Anaya/Instituto Cervantes. Recuperado de http://cvc.cervantes.es/ensenanza/biblioteca_ele/marco/

Corpas Jaime, García Eva, \& Agustín Garmendia (2003). Aula 1: curso de español. Barcelona: Difusión.

Cruz PiÑol, MAR (2015). Léxico y ELE: enseñanza/aprendizaje con tecnologías. Journal of Spanish Language Teaching, 2(2), 165-179.

DAvies, MARK (2006). A frequency dictionary of Spanish: Core vocabulary for learners. Nueva York: Routledge.

De Grève, Marcel, \& Van Passel, Frans (1971). Lingüística y enseñanza de lenguas extranjeras. Madrid: Fragua.

García Hoz, Víctor (1953). Vocabulario común, vocabulario usual y vocabulario fundamental. Madrid: Consejo Superior de Investigaciones Científicas.

Gutiérrez Cuadrado, Juan (Dir.) (1996). Diccionario Salamanca de la lengua española. Madrid: Santillana.

Ferrando Aramo, Verónica (2012). Aspectos teóricos y metodológicos para la compilación de un diccionario combinatorio destinado a estudiantes de E/LE (Tesis doctoral inédita). Tarragona: Universidad Rovira i Virgili.

Hidalgo, Andrea Fabiana (2009). Preparación al dele A1. Madrid: Edelsa.

Instituto Cervantes (2006). Plan Curricular del Instituto Cervantes. Niveles de referencia para el español. Recuperado de http://cvc.cervantes.es/ensenanza/biblioteca_ele/plan_curricular/ default.htm

Ji, RAN (2017). La enseñanza del léxico español del fútbol a estudiantes chinos de ELE (Tesis doctoral inédita). Tarragona: Universidad Rovira i Virgili.

Juilland, Alphonse G., \& Chang-Rodríguez, Eugenio (1964). Frequency dictionary of Spanish words. La Haya: Mouton.

Justicia Justicia, Fernando (1995). El desarrollo del vocabulario: diccionario de frecuencias. Granada: Universidad de Granada. 
López Morales, Humberto (1995). Los estudios de disponibilidad léxica: pasado y presente. Boletín de Filología de la Universidad de Chile, 35(1), 245-259.

López Morales, Humberto (Dir.) (s. f.). Dispolex. Recuperado de http://www.dispolex.com/info/ el-proyecto-panhispanico

López PÉrez, MARÍa ViCTORIa (2013). Criterios de selección de voces para la enseñanza del español como L2 en contextos escolares. Revista de Educación, 154-176.

Maldonado González, Concepción (Dir.) (1996). Clave. Diccionario de uso del español actual. Madrid: SM.

Maldonado González, Concepción (Dir.) (1999). Diccionario de sinónimos y antónimos. Madrid: SM.

McCullough, James L. (2001). Los usos de los córpora de textos en la enseñanza de lenguas. En Mireia Trenchs Parera (Coord.), Nuevas tecnologías para el autoaprendizaje y la didáctica de lenguas (pp. 125-140). Lleida: Milenio.

Márquez Villegas, Luis (1975). Vocabulario del español hablado: niveles y distribución gramatical. Madrid: Sociedad General Española de Librería.

Martín Peris, Ernesto, \& Neus Sans Baulenas (2004). Gente 1. Nueva edición. Barcelona: Difusión.

Ministère de L'ÉducATion NATiOnAle (1954). Le français fondamental ler degré. París: Institut Pédagogique National.

Paredes García, Florentino (2014). A vueltas con la selección de "centros de interés" en los estudios de disponibilidad léxica: para una propuesta renovadora a propósito de la disponibilidad léxica en ELE. Revista Nebrija de Lingüística Aplicada, 16, 54-59.

Real Academia Española (rae) (2005). Diccionario panhispánico de dudas. Recuperado de http://www.rae.es/

Real Academia Española (Rae) (2015). Diccionario de la lengua española. Recuperado de http://www.rae.es/

Real Academia Española (Rae). Banco de datos (CRea). Corpus de referencia del español actual. Recuperado de http://corpus.rae.es/creanet.html

Rodríguez Bou, Ismael (1952). Recuento de vocabulario español. Río Piedras: Consejo Superior de Enseñanza de Puerto Rico.

Rodríguez Muñoz, Francisco J., \& Muñoz Hernández, Isabel Ofelia (2009). De la disponibilidad a la didáctica léxica. Tejuelo: Didáctica de la Lengua y la Literatura. Educación, 4, 8-18. 
Rojo Sastre, Antonio José; Rivenc, Paul, \& Ferrer, Adam (1968). Vidas y diálogos de España. Primer grado. Lista alfabética de formas empleadas. Libro del profesor. París: Didier.

Salazar García, Ventura (2004). Acercamiento crítico a la selección objetiva de contenidos léxicos en la enseñanza de E/LE. Estudios de Lingüística Universidad de Alicante, 18, 243-273.

Sánchez, Aquilino; Sarmiento, Ramón; Cantos, Pascual, \& Simón, José (2001). Cumbre: Corpus lingüístico del español contemporáneo: fundamentos, metodología y aplicaciones. Madrid: Sociedad General Española de Librería.

Sánchez Lobato, Jesús, \& Acquaroni, Rosana (2014). Vocabulario ele B1: léxico fundamental de español de los niveles A1 a B1. Madrid: Sociedad General Española de Librería.

Santos Palmou, Xandra (2017). El vocabulario fundamental: historia, definición y nuevas propuestas aplicadas a la enseñanza de ELE. E-Aesla, 3, 110-120.

Scherfer, Peter. (1985). "Lexikalisches Lernen im Fremdsprachenunterricht". En Christoph Schwarze y Dieter Wunderlich (Eds.), Handbuch der Lexikologie (pp. 412-440). Kónigstein: Athenäum.

Terrádez Gurrea, Marcial (2001). Frecuencias léxicas del español coloquial: análisis cuantitativo y cualitativo. Valencia: Universidad de Valencia. (Cuadernos de Filología, XLI). 


\section{Apéndices}

\section{Apéndice 1. Nociones específicas de los manuales de ele sobre léxico de alimentación}

\begin{tabular}{|c|c|c|c|}
\hline Periodo & Manual & Palabras que ofrece en el nivel A1 & 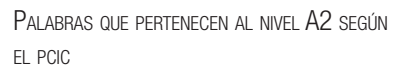 \\
\hline $\begin{array}{l}\text { Periodo } \\
2001-2004\end{array}$ & $\begin{array}{l}\text { Sueña } 1 \\
\text { (2001) }\end{array}$ & $\begin{array}{l}\text { almeja, arroz con leche, asado, barra, bollo, bo- } \\
\text { querón, calamar, chorizo, chuleta (de cerdo, de } \\
\text { ternera), crema catalana, fabada asturiana, flan, } \\
\text { gazpacho, mahonesa, mejillón, melón, nachos, } \\
\text { natillas, paella valenciana, pera, pimiento, pisto } \\
\text { manchego, puerro, refresco, salchicha, sopa de } \\
\text { marisco, tequila, tostada, ultramarinos, uva, vino } \\
\text { rosado }\end{array}$ & $\begin{array}{l}\text { aceite de oliva, ajo, arroz, atún, azúcar, } \\
\text { cava, cebolla, chocolate, filete de ter- } \\
\text { nera, galleta, gamba, harina, helado, } \\
\text { jamón, lechuga, mantequilla, manzana, } \\
\text { merluza, naranja, patata, plátano, pollo, } \\
\text { queso, ron, tomate, yogur, zanahoria, } \\
\text { zumo }\end{array}$ \\
\hline
\end{tabular}

Aula (2003) arroz a la cubana, arroz con leche, bistec con patatas, canelones, crema catalana, ensaladilla rusa, fabada, flan, gazpacho, huevos fritos con patatas, lentejas, melón, merluza a la romana, pescado (a la plancha, al vapor, asado, cocido, crudo, frito, guisado), sardinas a la plancha, tortilla (de patatas, española, francesa), verdura con patatas

Gente 1. Nueva aceitunas, ajo blanco, almendras, avellanas, caedición (2004)

Periodo Preparación al almorzar, almuerzo, banana, coliflor, condimen2009-2011 DELEA1 (2009) to, durazno, jugo, macarrones, melocotón, papa, plato único, vinagre, uva cliente, helado, jamón serrano, jamón york, mayonesa, menú del día, merluza, patatas, pollo, queso, salsas, yogur, zanahoria aceite de oliva, arroz, azúcar, carne de cerdo, carne de ternera, cava, cebollas, cereales, chocolate, cocinero, fresas, gambas, harina, jamón serrano, mantequilla, manzanas, naranjas, pasta, patatas, plátanos, pollo, queso, sal, tomates, yogur, zumo de naranja aceite, azúcar, jamón, la carta, lechuga, manzana, naranja, pasta, patata, pimienta, plátano, pollo, queso, sal, tomate, yogur, zumo

Nuevo ele ini- Pescador

cial 1 (2010) 
Hacia una propuesta de selección léxica en manuales de español como lengua extranjera de nivel A1 97

(continuación)

\begin{tabular}{|c|c|c|c|}
\hline Periodo & Manual & Palabras que ofrece en el nivel A1 & $\begin{array}{l}\text { PALABRAS QUe PERTENECEN AL NIVEL A2 SEGÚN } \\
\text { EL PCIC }\end{array}$ \\
\hline & $\begin{array}{l}\text { Bitácora } 1 \\
\text { (2011) }\end{array}$ & $\begin{array}{l}\text { almorzar, almuerzo, alubias, arepas, chorizo, } \\
\text { coca cola, comer fuera, cruasán, dulces, em- } \\
\text { butidos, empanadas, frutos secos, leche con } \\
\text { galletas, legumbres, maíz, marisco, montaditos, } \\
\text { ñoquis, pinchos, pizza, porotos, raviolis, taberna }\end{array}$ & $\begin{array}{l}\text { aceite de oliva, ajo, arroz, carne (de } \\
\text { cerdo, de cordero, de vaca, roja), ce- } \\
\text { reales, chocolate, comida rápida, pasta, } \\
\text { patatas, pollo, queso, tapa, tomar algo, } \\
\text { yogur, zumo }\end{array}$ \\
\hline \multirow[t]{2}{*}{$\begin{array}{l}\text { Periodo } \\
\text { 2013-2014 }\end{array}$} & $\begin{array}{l}\text { Nuevo Prisma } \\
\text { Nivel A1 } \\
(2013)\end{array}$ & $\begin{array}{l}\text { aceitunas, almorzar, almuerzo, antojitos, arepas, } \\
\text { bocaditos, bollería, boquerones en vinagre, bo- } \\
\text { tanas, calamares, chorizo, espaguetis, gazpa- } \\
\text { cho, lácteos, legumbres, limón, morcilla, paté, } \\
\text { pera, pulpo, refrescos, tapear, tortilla de patatas }\end{array}$ & $\begin{array}{l}\text { aceite de oliva, jamón, patatas, queso, } \\
\text { tapas, tomate, yogur, zumo }\end{array}$ \\
\hline & $\begin{array}{l}\text { Vocabulario } \\
\text { eLE B1: léxico } \\
\text { fundamental } \\
\text { de español de } \\
\text { los niveles A1 } \\
\text { a B1 } \\
\text { (2014) }\end{array}$ & $\begin{array}{l}\text { aceitunas, aperitivo, barra, berenjena, bizcocho, } \\
\text { boquerón, calabacín, calamar, caña, la carta, } \\
\text { cáscara, cereza, clara, cocinero, condimentos, } \\
\text { costilla, chorizo, chuleta, dieta, embutidos, es- } \\
\text { pecialidad, espinacas, garbanzos, guisantes, } \\
\text { harina, judías (blancas, verdes), kiwi, lácteos, } \\
\text { legumbres, lentejas, limón, lomo, magdalena, } \\
\text { manzanilla, margarita, marisco, melón, merme- } \\
\text { lada, miel, muslo, nata, pasteles, pechuga, pera, } \\
\text { piña, platos, poleo, propina, resaca, salchichón, } \\
\text { sandía, solomillo, tila, vino de la casa, yema }\end{array}$ & $\begin{array}{l}\text { aceite, ajo, aperitivo, atún, cebolla, cer- } \\
\text { do, champán, chocolate, cordero, filete, } \\
\text { galletas, gamba, ginebra, infusión, in- } \\
\text { grediente, jamón, lechuga, mantequilla, } \\
\text { manzana, merluza, naranja, patatas, } \\
\text { perejil, pimienta, plátano, pollo, queso, } \\
\text { receta, ron, sal, salmón, sardina, tapa, } \\
\text { tarta, ternera, tomate, yogur, zanahoria, } \\
\text { zumo }\end{array}$ \\
\hline
\end{tabular}

\section{Apéndice 2. Palabras de las nociones específicas del pcıc que no figuran en los manuales de ELE (A1)}

\begin{tabular}{|c|c|c|}
\hline PERIOdO & Manual & Palabras de LAS NOCIONES ESPECIFICAS DEL PCIC QUE NO FIGURAN EN EL MANUAL \\
\hline \multirow[t]{3}{*}{$\begin{array}{l}\text { Periodo } \\
2001-2004\end{array}$} & Sueña 1 (2001) & $\begin{array}{l}\text { agua mineral, bocadillo, café con leche, café cortado, café solo, carne, cena, cenar, co- } \\
\text { mer, comida, desayuno, ensalada, fruta, hamburguesa, la cuenta, merendar, merienda, } \\
\text { pescado, sándwich, té, tortilla }\end{array}$ \\
\hline & Aula (2003) & hamburguesa, mesa, leche, pan, sándwich, sopa \\
\hline & $\begin{array}{l}\text { Gente 1. Nueva } \\
\text { edición (2004) }\end{array}$ & hamburguesa, merienda, sándwich, sopa \\
\hline $\begin{array}{l}\text { Periodo } \\
\text { 2009-2011 }\end{array}$ & $\begin{array}{l}\text { Preparación al } \\
\text { DELEA1 (2009) }\end{array}$ & bar \\
\hline
\end{tabular}

Estudios de Lingüística Aplicada, año 36, número 68, diciembre de 2018, pp. 59-98 doi: 10.22201/enallt.01852647p.2018.68.744 
98 Ángel Huete García y Antoni Nomdedeu Rull

(continuación)

\begin{tabular}{|c|c|c|}
\hline PeRIODO & MANUAL & PaLABRAS DE LAS NOCIONES ESPECIFICAS DEL PCIC QUE NO FIGURAN EN EL MANUAL \\
\hline \multirow{4}{*}{$\begin{array}{l}\text { Periodo } \\
\text { 2013-2014 }\end{array}$} & $\begin{array}{l}\text { Nuevo ELE inicial } 1 \\
\text { (2010) }\end{array}$ & $\begin{array}{l}\text { bocadillo, cena, carne, desayuno, el menú, ensalada, fruta, hamburguesa, huevos, me- } \\
\text { rendar, merienda, paella, pan, pescado, postre, primer/segundo plato, sándwich, sopa, } \\
\text { tortilla, verdura }\end{array}$ \\
\hline & Bitácora 1 (2011) & paella, sándwich \\
\hline & $\begin{array}{l}\text { Nuevo Prisma } \\
\text { Nivel A1 (2013) }\end{array}$ & $\begin{array}{l}\text { beber, cerveza, comer, desayunar, el menú, ensalada, merendar, pan, primer/segundo } \\
\text { plato, sándwich, vino }\end{array}$ \\
\hline & $\begin{array}{l}\text { Vocabulario } \\
\text { ELE B1: léxico } \\
\text { fundamental de } \\
\text { español de los } \\
\text { niveles A1 a B1 } \\
\text { (2014) }\end{array}$ & bocadillo, hamburguesa, la cuenta, sándwich \\
\hline
\end{tabular}

Estudios de Lingüística Aplicada, año 36, número 68, diciembre de 2018, pp. 59-98

doi: 10.22201/enallt.01852647p.2018.68.744 